\title{
Long-term results of the mucosal ablation of Barrett's esophagus: efficacy and recurrence
}

Authors

Institutions
Shreyas Saligram ${ }^{1,2}$, Nathan Tofteland ${ }^{1,2}$, Sachin Wani ${ }^{3}$, Neil Gupta $^{4}$, Sharath Mathur ${ }^{1}$, Prashanth Vennalaganti ${ }^{1}$, Vijay Kanakadandi $^{1,2}$, Maria Giacchino ${ }^{1}$, April Higbee ${ }^{1}$, Diego Lim ${ }^{1,2}$, Amit Rastogi ${ }^{1,2}$, Ajay Bansal ${ }^{1,2}$, Prateek Sharma $^{1,2}$ Institutions are listed at the end of article. submitted

19. October 2014 accepted after revision 31. October 2014

\section{Bibliography}

DOI http://dx.doi.org/

10.1055/s-0034-1391669

Published online: 7.5.2015

Endosc Int Open 2015; 03:

E189-E194

(c) Georg Thieme Verlag KG Stuttgart $\cdot$ New York

E-ISSN 2196-9736

\section{Corresponding author}

\section{Prateek Sharma, MD}

Department of

Gastroenterology (111)

Veterans Affairs Medical Center 4801 East Linwood Boulevard Kansas City, Missouri 64128 2295

Fax: +1-412-708-5548

psharma@kumc.edu
Background and study aims: It has been postulated that the endoscopic ablation of Barrett's esophagus can lead to complete eradication of the disease. This study was undertaken to evaluate the efficacy of endoscopic eradication therapy for Barrett's esophagus and the rates of recurrence of intestinal metaplasia.

Patients and methods: As part of an initial randomized controlled trial, patients with nondysplastic or low grade dysplastic Barrett's esophagus underwent mucosal ablation. Following ablation, the patients had annual surveillance endoscopies. Recurrence was defined as the presence of intestinal metaplasia after initial complete eradication had been achieved.

Results: A total of 28 patients with Barrett's esophagus were followed for a mean of 6.4 years after ablation therapy. At baseline, the majority of the patients had nondysplastic Barrett's esophagus (79\%). Initial complete eradication of intestinal metaplasia was achieved at a mean of 4.1

\section{Introduction}

\section{$\nabla$}

Barrett's esophagus is defined as replacement of the normal squamous epithelium of the tubular esophagus by intestinal metaplasia in a region proximal to the gastroesophageal junction (GEJ) [1]. Barrett's esophagus is the only established premalignant condition for esophageal adenocarcinoma (EAC), a cancer with a dismal 5-year survival rate. EAC has been observed to be the most rapidly increasing cancer in the United States [2]; its annual incidence is reported to be between $0.12 \%$ and $0.5 \%$ in individuals with Barrett's esophagus [3-5].

Intestinal metaplasia can exhibit histologic transformation through stages of low grade dysplasia and high grade dysplasia to EAC [6]. The high rate of progression of high grade dysplasia to EAC without treatment is well described [7]. The reported incidence of high grade dysplasia arising months. During long-term follow-up, initial recurrence of intestinal metaplasia was seen in 14 of the 28 of patients (50\%) at a mean of 40 months, and further maintenance ablation therapy was applied. At the final follow-up, 36\% of the patients had complete eradication of intestinal metaplasia, $18 \%$ of the patients had intestinal metaplasia, and $21 \%$ had died of unrelated causes; invasive esophageal adenocarcinoma had developed in 1 patient.

Conclusions: The long-term results of this study demonstrate a recurrence rate of $50 \%$ after complete eradication of Barrett's esophagus with endoscopic eradication therapy. In addition, rerecurrence (in 36\%), even after further maintenance endoscopic eradication therapy, and deaths unrelated to the disease (21\%) occurred. Complete remission of Barrett's esophagus appears to be a difficult goal to achieve. These results call into question the role of ablation in patients with low risk Barrett's esophagus.

from nondysplastic Barrett's esophagus is 0.48\% per year and from low grade dysplastic Barrett's esophagus is $1.6 \%$ per year $[8,9]$. In contrast, the risk of EAC developing from nondysplastic Barrett's esophagus is $0.2 \%$ per year and from low grade dysplastic Barrett's esophagus is $0.44 \%$ per year [5, $8-10]$. Some have cited higher figures for the progression of low grade dysplasia to high grade dysplasia and EAC (up to $13.6 \%$ per year) $[11,12]$.

Several investigators have suggested that the endoscopic ablation of nondysplastic and low grade dysplastic Barrett's esophagus could lead to the complete eradication of the disease and potentially prevent the progression to cancer. The recent use of radiofrequency ablation (RFA) in this patient population has yielded encouraging short-term results. However, data for the longterm results of the treatment of nondysplastic and low grade dysplastic Barrett's esophagus are 


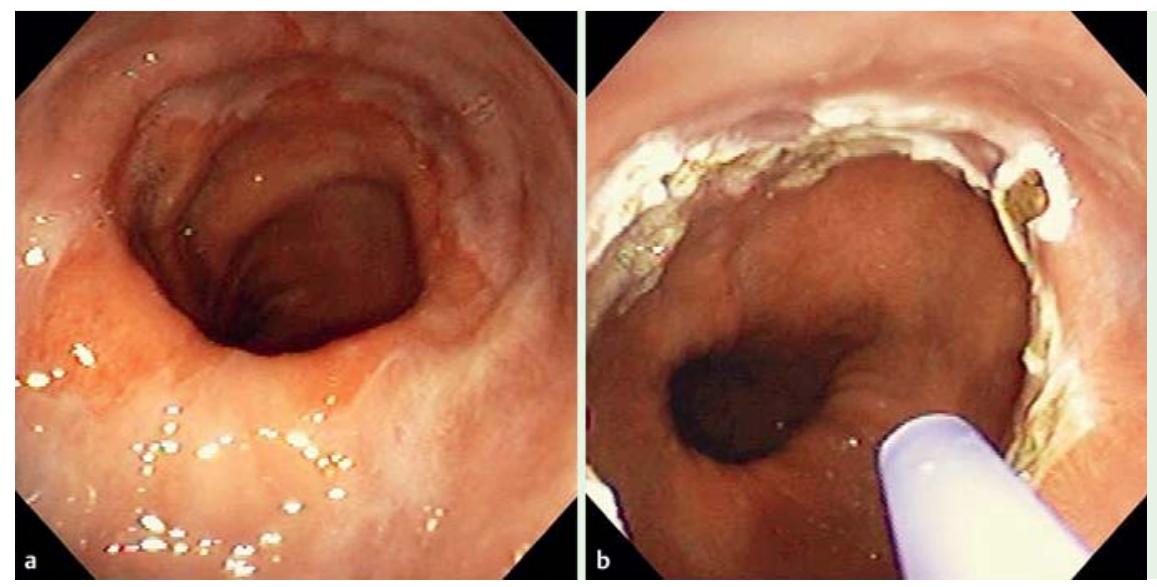

Fig. 1 Barrett's esophagus (a) before and (b) after argon plasma coagulation.

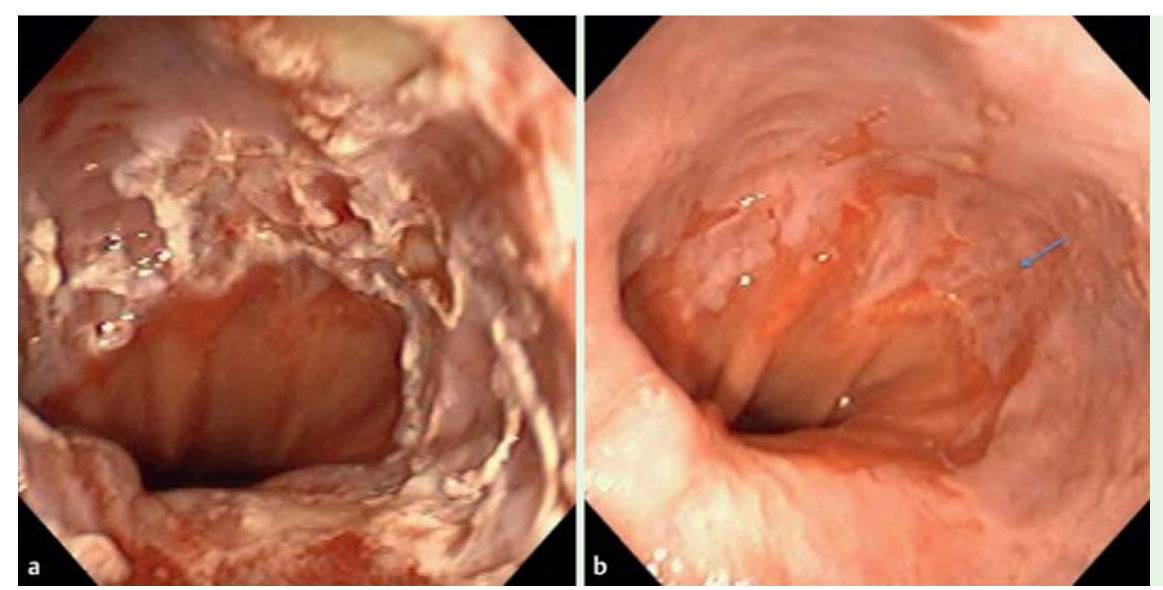

Fig. 2 a Ablation of Barrett's esophagus with multipolar electrocoagulation. b Arrow indicates region of recurrence of columnar metaplasia after complete ablation.

limited. Furthermore, the rate of re-recurrence of Barrett's esophagus after continued maintenance ablation therapy has not been reported.

We previously conducted a randomized controlled trial comparing the short-term efficacy of argon plasma coagulation (APC) and that of multipolar electrocoagulation (MPEC) in patients with Barrett's esophagus. In this report, we present the longterm efficacy and outcomes in this group of patients with nondysplastic and low grade dysplastic Barrett's esophagus.

\section{Patients and methods \\ $\nabla$}

\section{Patients and endoscopic therapy}

A total of 35 patients with established Barrett's esophagus (both nondysplastic and low grade dysplastic Barrett's esophagus) underwent stratified randomization based on the length of the affected segment to undergo endoscopic eradication therapy with APC or MPEC as part of a randomized controlled trial. This was done to evaluate the two techniques of APC and MPEC. Details of the initial randomized controlled trial have previously been published [13]. Briefly, patients underwent endoscopic eradication therapy with either APC or MPEC every 4 to 8 weeks until complete eradication of intestinal metaplasia (CE-IM) or a maximum of 6 treatment sessions ( $\bullet$ Fig. 1 and $\bullet$ Fig. 2). The study was approved by the local institutional review board.

\section{Definitions and surveillance endoscopy}

Barrett's esophagus was defined as the presence of columnar epithelium lining the esophagus and intestinal metaplasia on biopsy specimens. The top of the gastric folds was considered the anatomical GEJ; biopsy specimens obtained above this landmark represented those from the tubular esophagus.

Following endoscopic eradication therapy, all patients underwent interval surveillance endoscopies every 6 months for the first year and annually thereafter per the study protocol. Narrow-band imaging was used to examine the length of the previously diseased esophagus to evaluate for columnar mucosa. Four-quadrant biopsy specimens were obtained from visible columnar epithelium at intervals of 1 to $2 \mathrm{~cm}$. If there was no evidence of visible columnar epithelium, four-quadrant biopsy specimens were obtained from the length of the previously diseased esophagus at intervals of 1 to $2 \mathrm{~cm}$. Specimens were also obtained from the cardia per the study protocol. They were placed in separate containers. An experienced gastrointestinal pathologist assessed the biopsy specimens.

CE-IM was defined as the absence of columnar epithelium lining the tubular esophagus on endoscopy and surveillance biopsy specimens obtained from the length of the previously diseased esophagus showing no evidence of intestinal metaplasia.

\section{Recurrences and management}

Recurrence of Barrett's esophagus was defined as the identification on endoscopy of columnar epithelium / characteristic salmon-pink tongues in the tubular esophagus above the GEJ, with surveillance biopsy specimens of the epithelium showing evi- 

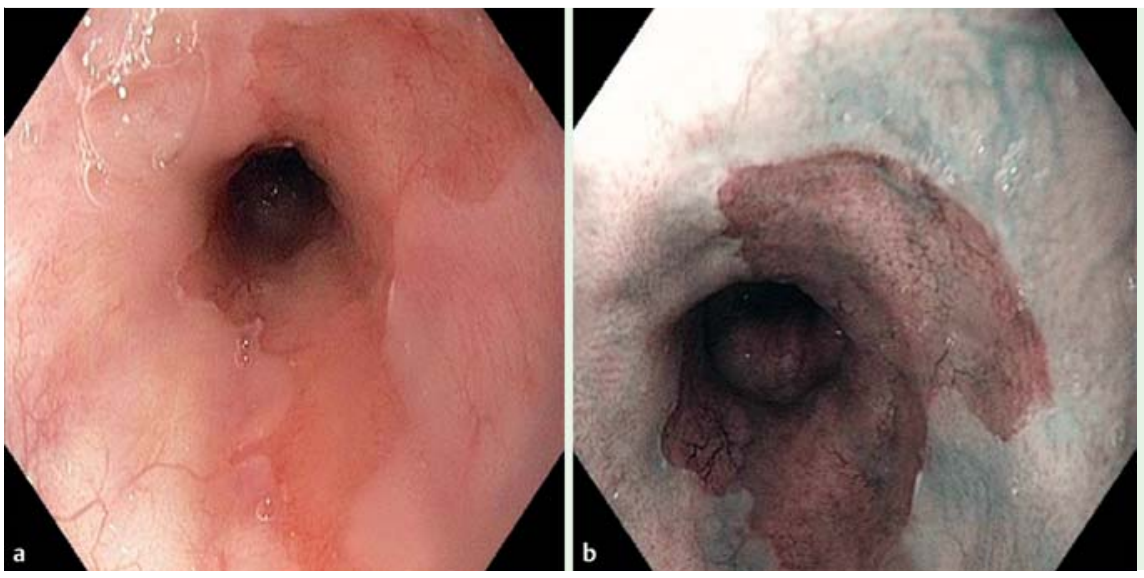

Fig. 3 Recurrence of Barrett's esophagus (a) in white light and (b) on narrow-band imaging.

dence of intestinal metaplasia ( Fig.3). Any evidence of intestinal metaplasia in the cardia was not considered a histologic recurrence.

The presence of any columnar epithelium, including an irregular $\mathrm{Z}$ line with extensions above the GEJ, was treated with mucosal ablation. Any evidence of intestinal metaplasia in the cardia was monitored with surveillance endoscopy and not treated with endoscopic eradication therapy.

\section{Data collection and analysis}

Pertinent information regarding demographics, endoscopic findings, eradication method, number of ablation sessions, surveillance biopsy findings, recurrence of Barrett's esophagus (intestinal metaplasia and dysplasia), and maintenance endoscopic eradication therapy sessions was collected from the electronic medical records. Medical management after ablation and longterm response to endoscopic eradication therapy were assessed by review of the clinical records. Recurrence was defined as the presence of intestinal metaplasia after initial complete eradication had been achieved. Recurrence of Barrett's esophagus was treated with maintenance APC ablation. The duration of followup was calculated from the time of the last endoscopic treatment in the randomized controlled trial.

Normally distributed variables were summarized as mean and standard deviation (SD). Variables with a skewed distribution were reported as median and interquartile range (IQR). Percentages were used to report categorical variables.

\section{Results}

Patient details and initial response to ablation:

A total of 28 patients were included in this long-term follow-up study. All patients were male and $96 \%$ were Caucasian. At enrollment, the mean (SD) age was 59.5 years (7.7). The mean (SD) length of Barrett's esophagus was $3.5 \mathrm{~cm}$ (1.6). Hiatus hernia was found in $93 \%$ of patients with a mean (SD) size of $2.5 \mathrm{~cm}(0.9)$. At baseline, 22 of the patients (79\%) had nondysplastic Barrett's esophagus and 6 of the patients (21\%) had low-grade dysplasia. All histologic findings were confirmed by an experienced gastrointestinal pathologist.

Following endoscopic eradication therapy (13 APC, 15 MPEC), all patients achieved CE-IM at a median of 4.1 months (IQR 1-13). The mean number of endoscopic eradication therapy sessions to achieve initial CE-IM was 3.3 (3.2 MPEC, 3.5 APC). The initial
MPEC or APC treatment did not fail in any patient, and no patient was excluded from the series because of failure of treatment.

\section{Long-term follow-up and maintenance ablation}

The patients were followed for a mean of 6.4 years (SD 3.4 and IQR 11-141 months) after CE-IM. The mean number of followup endoscopies was 6.2 (IQR 2-12). The total number of patients lost to follow-up was 9. Excluding the patients who were lost to follow-up, the mean follow-up was 92 months (7.7 years; IQR 11-141 months). Excluding the patients who were lost to follow-up, the mean number of follow-up endoscopies was 7.2 (IQR 2-12). During long-term follow-up, recurrence of intestinal metaplasia was seen in 14 patients (50\%) at a mean of 40 months (IQR $11-110)$. Of the 14 patients with recurrent intestinal metaplasia, 10 (71\%) subsequently achieved CE-IM after further maintenance endoscopic eradication therapy (mean of 1.4 additional sessions). However, 3 of the 10 patients with a second CE-IM (30\%) had re-recurrence of intestinal metaplasia at an average of 88 months (IQR 49-123). Of these 3 patients with re-recurrence, $2(67 \%)$ had persistent intestinal metaplasia at the most recent endoscopic follow-up. The baseline diagnoses in the 14 patients with recurrence were nondysplastic Barrett's esophagus in 10 patients and low grade dysplasia in 4 patients. None of the patients had complete recurrence of the original Barrett's segment. All recurrences were in the distal esophagus; endoscopically, they appeared as small islands/tongues of columnar mucosa above the GEJ with intestinal metaplasia on biopsy specimens. No patient had buried intestinal metaplasia on surveillance endoscopy after endoscopic eradication therapy.

Flowchart ( $\bullet$ Fig. 4) tracks the outcomes of the patients followed in the study. At the final follow-up, 10 of the 28 patients had CEIM (36\%). A Kaplan-Meier curve for the recurrence of intestinal metaplasia is shown in $\bullet$ Fig. 5 . There were 5 patients with persistent intestinal metaplasia. There were 2 patients with persistent intestinal metaplasia despite maintenance endoscopic eradication therapy, and 1 of them was lost to follow-up after being followed for 100 months. There were 3 patients with persistent intestinal metaplasia who did not undergo maintenance endoscopic eradication therapy. Of these, 1 patient chose not to undergo further endoscopic eradication therapy and is undergoing regular surveillance endoscopies, 1 patient died of causes other than Barrett's esophagus, and 1 patient was lost to follow-up early in the study. During the follow-up period, 6 patients (21\%) died of causes other than Barrett's esophagus/esophageal cancer. 
28 patients (15 MPEC and $13 \mathrm{APC})$

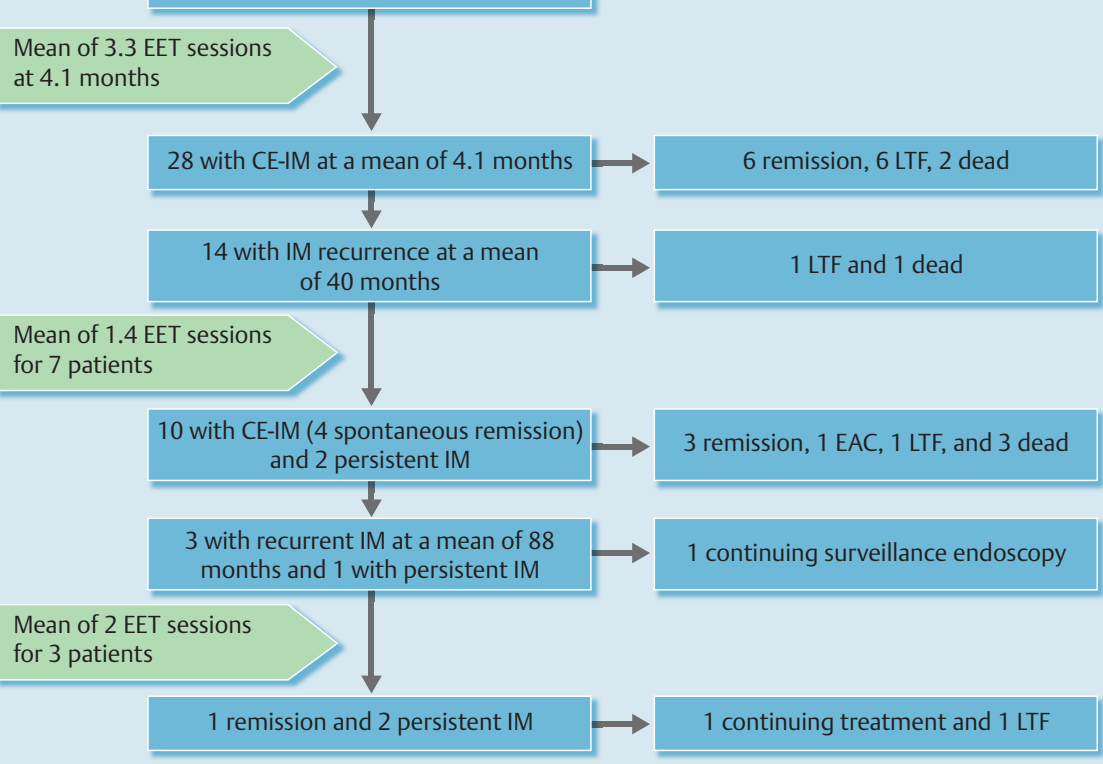

Fig.4 Treatment outcomes of mucosal ablation of Barrett's esophagus.
Intestinal metaplasia of the cardia

Of the 28 patients, 5 (18\%) had intestinal metaplasia in the cardia on surveillance endoscopy. The baseline histology of these $5 \mathrm{pa}-$ tients before commencing endoscopic eradication therapy was nondysplastic Barrett's esophagus in 4 patients and low grade dysplasia in 1 patient. Of these 5 patients, 3 also had histologic recurrence of Barrett's esophagus, with intestinal metaplasia diagnosed by surveillance endoscopy from the biopsy specimens obtained above the GEJ.

\section{Cancer development}

EAC developed in 1 patient (3.6\%). After initial CE-IM, the patient was noted to have an irregular $Z$ line, and intestinal metaplasia initially recurred 28 months after the completion of endoscopic eradication therapy. Subsequent surveillance biopsies continued to show persistent intestinal metaplasia. Unfortunately, this patient was lost to follow-up for surveillance endoscopy for 3 years. He finally presented for surveillance endoscopy, and EAC was diagnosed 9.4 years after he had achieved CE-IM. He subsequently underwent esophagectomy, and a T1bN0 cancer was diagnosed. There was evidence of recurrence of intestinal metaplasia during his surveillance after surgery that required 1 session of APC, but since then, there has been no recurrence of intestinal metaplasia.

\section{Discussion \\ $\nabla$}

Although endoscopic ablation therapy for Barrett's esophagus has been used for the past several years, the long-term followup of eradication and recurrence, the role of maintenance ablation therapy, and re-recurrences after continued ablation are not clear [14]. Given the possibility of the progression of nondysplastic and low grade dysplastic Barrett's esophagus, it has been postulated that endoscopic therapy of these lesions could lead to complete eradication of the disease with the prevention of neoplastic progression. In fact, several short-term studies of patients with nondysplastic Barrett's esophagus have shown promising eradication rates with the use of RFA, APC, and MPEC.
Results of this study, which included long-term follow-up of patients from a previously conducted randomized control trial comparing APC and MPEC, showed that $50 \%$ of patients had recurrence of intestinal metaplasia at 40 months. Nearly half of these patients with recurrence underwent further endoscopic eradication therapy, with $71 \%$ of the patients again achieving CE-IM at a mean of 53 months; on average, 1 session of maintenance ablation was required every 3 to 4 years. However, $30 \%$ of the patients in remission developed a second recurrence of intestinal metaplasia at a mean of 88 months. Persistent intestinal metaplasia was noted in 5 patients at a mean follow-up of 6.4 years, and EAC was diagnosed in 1 patient.

Some of the earlier published studies also reported eradication and recurrence rates of nondysplastic and low grade dysplastic Barrett's esophagus in patients undergoing APC and MPEC ablation therapy. An observational study with a long-term follow-up of 10 years of 139 patients who underwent endoscopic eradication therapy with MPEC for nondysplastic Barrett's esophagus revealed that all patients achieved CE-IM after a mean of 2.8 ablation sessions. However, $5 \%$ had a recurrence of intestinal meta-

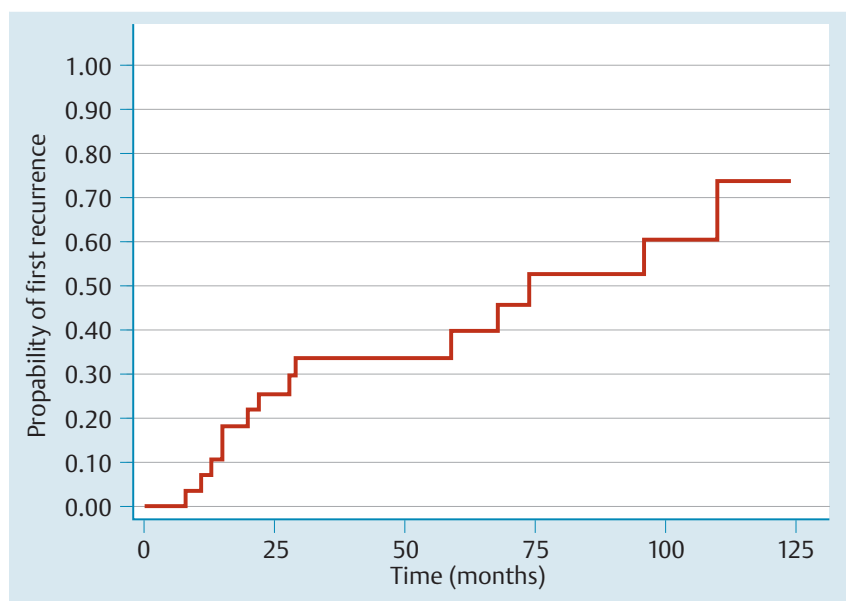

Fig. 5 Kaplan-Meier curve for the recurrence of intestinal metaplasia. 
plasia within 2 years of treatment [15]. Another prospective study included 21 patients treated with APC for nondysplastic Barrett's esophagus and followed for a mean of 30 months. Although all patients achieved CE-IM initially after completion of treatment, there was evidence of recurrence of intestinal metaplasia in $66 \%$ of the patients after a mean of 15.5 months that required maintenance APC sessions. CE-IM was achieved in only $33 \%$ of the patients at the end of final follow-up [16]. More recently, 59 patients with either nondysplastic or low grade dysplastic Barrett's esophagus were randomized either to receive APC treatment or to undergo surveillance without treatment. Complete endoscopic and histologic eradication was seen in 19\% of the patients in the treatment group and in $7 \%$ in surveillance group after 7 years of follow-up [17].

Furthermore, $18 \%$ of patients in this cohort had intestinal metaplasia in the cardia after ablation. This finding has been reported by other investigators as well, who observed intestinal metaplasia in the area of the GEJ after ablation. It is unclear if this finding can lead to dysplasia or adenocarcinoma in the esophagus [18].

Since we started using APC and MPEC for ablation, endoscopic eradication therapy has undergone significant change in the last decade. RFA is the best-studied endoscopic eradication therapy that is currently available [19]. A prospective multicenter trial looking at the durability of RFA treatment in 50 patients with nondysplastic Barrett's esophagus showed that all patients achieved CE-IM in 1 year. A 5-year follow-up revealed recurrence of intestinal metaplasia in $8 \%$ of the patients, who required further maintenance RFA sessions [20]. However, other studies of RFA have reported recurrence rates of up to $33 \%$ at 2-year follow-up [21, 22]. Therefore, long-term follow-up of RFA requires continued surveillance endoscopies and maintenance endoscopic eradication therapy.

The strength of this study is the duration of follow-up after endoscopic eradication therapy, providing findings that contribute to the growing body of literature on long-term outcomes following such treatment. The study also has several limitations. Only a limited number of patients were followed long term, and therapy was performed at a single center. Another drawback is that ablation therapy with APC or MPEC was used, which is not currently offered in many centers and has been primarily replaced by RFA therapy. However, this should not be a major concern because the fundamental principles of endoscopic eradication therapy remain the same, and as long-term follow-up after RFA is becoming available, recurrences are being reported. One hypothesis concerning recurrence is that the recurrence of Barrett's esophagus is linked to the persistence of gastroesophageal reflux disease. Patients were prescribed twice-daily proton pump inhibitors, but compliance was not checked and $\mathrm{pH}$ studies were not performed. Therefore, we do not have any idea whether ongoing reflux contributed to the recurrences. Perhaps better compliance with long-term proton pump inhibitor therapy could reduce the recurrence of Barrett's esophagus. However, this issue must be explored in future trials.

In conclusion, although short-term benefits may be seen with ablation in patients with nondysplastic Barrett's esophagus, the long-term results of this study demonstrate a recurrence rate of $50 \%$ after complete eradication of Barrett's esophagus with endoscopic eradication therapy. In addition, re-recurrence (in 36\%), even after further maintenance endoscopic eradication therapy, is possible. Up to $30 \%$ of patients require maintenance ablation sessions (approximately 1 ablation session every 3 to 4 years) to keep the disease in remission. Finally, deaths unrelated to the dis- ease $(21 \%)$ can occur during follow up.Complete remission of Barrett's esophagus appears to be a difficult goal to achieve. These results call into question the role of ablation therapy in patients with low risk Barrett's esophagus.

Competing interests: Dr. Sharma has received grant support from Barrx Medical, Cook Medical, NinePoint Medical, and Olympus. The other authors have no conflicts of interest to declare.

\section{Institutions}

${ }^{1}$ Veterans Affairs Medical Center, Kansas City, Missouri, USA

2 University of Kansas School of Medicine, Kansas City, Kansas, USA

${ }^{3}$ University of Colorado and Veterans Affairs Medical Center, Denver,

Colorado, USA

${ }^{4}$ Loyola University Health System, Maywood, Illinois, USA

\section{References}

1 Wang KK, Sampliner RE. Updated guidelines 2008 for the diagnosis, surveillance and therapy of Barrett's esophagus. Am J Gastroenterol 2008; 103: 788-797

2 Pohl H, Welch HG. The role of overdiagnosis and reclassification in the marked increase of esophageal adenocarcinoma incidence. J Natl Cancer Inst 2005; 97: 142 - 146

3 de Jonge PJF, van Blankenstein M, Looman CWN et al. Risk of malignant progression in patients with Barrett's oesophagus: a Dutch nationwide cohort study. Gut 2010; 59: 1030-1036

4 Bhat S, Coleman HG, Yousef F et al. Risk of malignant progression in Barrett's esophagus patients: results from a large population-based study. J Natl Cancer Inst 2011; 103: 1049-1057

5 Hvid-Jensen F, Pedersen L, Drewes AM et al. Incidence of adenocarcinoma among patients with Barrett's esophagus. N Engl J Med 2011; 365: $1375-1383$

6 Schlemper RJ, Riddell RH, Kato Y et al. The Vienna classification of gastrointestinal epithelial neoplasia. Gut 2000; 47: 251 -255

7 Rastogi A, Puli S, El-Serag HB et al. Incidence of esophageal adenocarcinoma in patients with Barrett's esophagus and high-grade dysplasia: a meta-analysis. Gastrointest Endosc 2008; 67: 394-398

8 Wani S, Falk G, Hall $M$ et al. Patients with nondysplastic Barrett's esophagus have low risks for developing dysplasia or esophageal adenocarcinoma. Clin Gastroenterol Hepatol 2011; 9: 220-227.e1

9 Gaddam S, Singh M, Balasubramanian G et al. Persistence of nondysplastic Barrett's esophagus identifies patients at lower risk for esophageal adenocarcinoma: results from a large multicenter cohort. Gastroenterology 2013; 145: 548-553.e1

10 Wani S, Falk GW, Post $J$ et al. Risk factors for progression of low-grade dysplasia in patients with Barrett's esophagus. Gastroenterology 2011; 141: 1179-1186.e1

11 Curvers WL, ten Kate FJ, Krishnadath KK et al. Low-grade dysplasia in Barrett's esophagus: overdiagnosed and underestimated. Am J Gastroenterol 2010; 105: 1523 - 1530

12 Phoa KN, van Vilsteren FG, Weusten BL et al. Radiofrequency ablation vs endoscopic surveillance for patients with Barrett esophagus and lowgrade dysplasia: a randomized clinical trial. JAMA 2014; 311: 12091217

13 Sharma P, Wani S, Weston AP et al. A randomised controlled trial of ablation of Barrett's oesophagus with multipolar electrocoagulation versus argon plasma coagulation in combination with acid suppression: long-term results. Gut 2006; 55: 1233-1239

14 Sharma P. Clinical practice. Barrett's esophagus. N Engl J Med 2009; 361: $2548-2556$

15 Allison $H$, Banchs MA, Bonis PA et al. Long-term remission of nondysplastic Barrett's esophagus after multipolar electrocoagulation ablation: report of 139 patients with 10 years of follow-up. Gastrointest Endosc 2011; 73: $651-658$

16 Mork H, Al-Taie O, Berlin F et al. High recurrence rate of Barrett's epithelium during long-term follow-up after argon plasma coagulation. Scand J Gastroenterol 2007; 42: $23-27$

17 Sie C, Bright T, Schoeman M et al. Argon plasma coagulation ablation versus endoscopic surveillance of Barrett's esophagus: late outcomes from two randomized trials. Endoscopy 2013; 45: 859-865

18 Pascarenco OD, Boeriu A, Mocan S et al. Barrett's esophagus and intestinal metaplasia of gastric cardia: prevalence, clinical, endoscopic and histological features. J Gastrointestin Liver Dis 2014; 23: 19-25 
19 Saligram S, Chennat J, Hu H et al. Endotherapy for superficial adenocarcinoma of the esophagus: an American experience. Gastrointest Endosc 2013; 77: $872-876$

20 Fleischer DE, Overholt BF, Sharma VK et al. Endoscopic radiofrequency ablation for Barrett's esophagus: 5-year outcomes from a prospective multicenter trial. Endoscopy 2010; 42: 781-789

21 Haidry RJ, Dunn JM, Butt MA et al. Radiofrequency ablation and endoscopic mucosal resection for dysplastic Barrett's esophagus and early esophageal adenocarcinoma: outcomes of the UK National Halo RFA Registry. Gastroenterology 2013; 145: 87-95

22 Gupta M, Iyer PG, Lutzke $L$ et al. Recurrence of esophageal intestinal metaplasia after endoscopic mucosal resection and radiofrequency ablation of Barrett's esophagus: results from a US Multicenter Consortium. Gastroenterology 2013; 145: 79-86 